\title{
Target Dependence of Adult Neurons: Pattern of Terminal Arborizations
}

\author{
S. Marty, J. M. Weinitz, and M. Peschanski \\ INSERM CJF 91-02, Faculté de médecine, 94010 Créteil, France
}

During development, the survival of neurons in the CNS depends critically on interactions with postsynaptic target cells. The role of target celis on the maintenance of afferent neurons in the adult, however, is a matter of controversy. Morphological alterations of target-deprived neurons, such as axonal retraction or pruning, may occur. We have therefore undertaken an analysis of target-deprived neurons over time after an excitotoxic lesion in order to investigate these potential changes.

Dorsal column nuclei (DCN) neurons were deprived of their target neurons in adult rats by the injection of kainic acid into the ventrobasal thalamic complex. Anterogradely transported wheat germ agglutinin conjugated to HRP from the DCN showed a progressive decrease of the density of afferent terminals during the first month after lesion. Density stabilized thereafter through the longest time studied (8 months). In contrast, the extent of the area occupied by DCN projections did not change up to 1 month and then shrank over time. These results indicated a continuous decrease in the number of axonal elements in the lesion, which is the strongest during the first month postlesion.

To interpret these data, we then studied axonal morphology. Diffusion-filled lemniscal axons were labeled by WGAHRP injections aimed at the medial lemniscus. There was no conspicuous alteration of axonal stems in the medial lemniscus. Terminal axonal arborizations and swellings dramatically decreased in number over the first month after the kainate injection, and large axonal varicosities were formed over the same period of time. These morphological data suggest that the decrease in number of axons in an excitotoxic lesion is related, at least during the first month, to a loss of axonal terminal arborizations rather than to a retraction of axonal stems.

The pattern of terminal arborizations in the adult CNS may therefore depend critically on interactions of afferents with their target neurons, while the maintenance of the axonal stems does not.

[Key words: excitotoxic lesion, axonal arbor, pruning, axonal varicosity, rat, postsynaptic dependence]

\footnotetext{
Received Nov. 8, 1993; revised Feb. 10, 1994; accepted Feb. 24, 1994.

We thank Dr. Isabelle Dusart and Dr. Salvatore Rapisardi for their critical review of the manuscript. This work was supported by Ministère de la Recherche et de la Technologie (MRT 106728).

Correspondence should be addressed to M. Peschanski, INSERM CJF 91-02, Faculté de médecinc, 8 ruc de génćral Sarrail, 94010 Créteil, France.

Copyright (C) 1994 Society for Neuroscience 0270-6474/94/145257-10\$05.00/0
}

During development, the survival of neurons in the CNS depends critically upon interactions with postsynaptic target cells (reviewed in Cowan et al., 1984). In the aduit CNS the existence of such interactions is a matter of controversy. Axotomized neurons that are deprived of any interaction with their targets have been shown to die, but the relative roles of the lesion and the loss of postsynaptic influence are not clearly established (Sofroniew and Isacson, 1988; Villegas-Pérez et al., 1993). Excitotoxins injected in the adult CNS kill neurons while acutely sparing afferent fibers. They thus appear to be good tools for the study of target deprivation. Results obtained with these techniques are, however, controversial. Recent studies in which septohippocampal neurons were deprived of target neurons by an excitotoxic lesion failed to reveal a loss of afferent neurons (Sofroniew et al., 1990; Kordower et al., 1992). In contrast, retinal ganglion cells degenerated after neuronal depletion in the dorsal-lateral geniculate body (Pearson and Stoffler, 1992). Degeneration of afferent neurons, however, occurred months after target deprivation.

Morphological alterations of afferent neurons related to target deprivation, such as axonal retraction or pruning, have not been explored in similar detail, even though these potential changes may provide clues about the way target cells influence afferent neurons. Many studies considering afferent axons to an excitotoxically lesioned area have indicated a decrease in axonal density over time (Zaczek et al., 1978; Peschanski and Besson, 1987; Pearson et al., 1992). Coyle and Schwarcz (1983) hypothetized that afferent axons deprived of target neurons retracted. This has not been clearly established, however, and an alternative hypothesis could be a constriction of the axonal terminal field without actual retraction of axonal stems. The fact, for instance, that dopamine in the excitotoxically lesioned striatum decreases in concentration (Isacson et al., 1985) while dopaminergic neurons can still all be labeled retrogradely from the same site (Wictorin et al., 1988) argues in favor of the latter hypothesis. A loss of terminal arborizations of climbing fibers after excitotoxic lesion of Purkinje cells has been demonstrated (Armengol et al., 1989; Rossi et al., 1993). Morphological alterations of afferent axons have been described after excitotoxic lesions to various other structures of the brain (Meibach et al., 1978; Walker and McAllister, 1986; Peschanski and Besson, 1987; Nothias et al., 1988; Pearson et al., 1992). Axons present enlargements characterized by an abundance of mitochondria, microtubules, and lysosomes (Peschanski and Besson, 1987). Whether these alterations accompany retraction or pruning of afferents remains to be shown. The present set of studies has been designed specifically to analyze morphological changes of lemniscal afferents deprived of their target neurons in the ven- 
trobasal thalamic complex, in relation to changes in the number of axons in the lesion.

The results suggest that, at least during the first month, the decrease in the number of axons occurring in the lesion is not accompanied by a retraction of axonal stems but rather is due to a dramatic loss of axonal terminal arborizations. This suggests a specific role of postsynaptic target neurons upon the maintenance of this terminal axonal compartment.

\section{Materials and Methods}

A total of 38 female Sprague-Dawley rats (Charles River; 200-250 gm at the beginning of the experiments) was used in these studies.

Lesioning procedure. The rats were anesthetized (chloral hydrate, 400 $\mathrm{mg} / \mathrm{kg}$, i.p.) and received a slow unilateral pressure injection of either $\mathrm{KA}$ ( $5 \mathrm{nmol}$ in $0.15 \mu \mathrm{l}$ saline, over $12 \mathrm{~min})(n=36)$ or saline ("sham," $n=2$ ) using a $1 \mu \mathrm{l}$ Hamilton syringe. Injections were aimed at the internal capsule at the border of the rostral thalamus (Peschanski and Besson, 1987), in order to produce by diffusion of KA a large neuronal loss in the right thalamus, while avoiding mechanical lesioning of the DCN afferents in VB. Animals were supplemented with a $5 \%$ glucose saline solution ( $10 \mathrm{cc}$ daily) for $5 \mathrm{~d}$ to limit the effects of an observed transient adipsic-aphagic statc (Divac ct al., 1978).

Anterograde labeling of $D C N$ projections with $W G A-H R P$. At 4,9 , 16 , and $23 \mathrm{~d}$ and $1,2,4,6$, and 8 months after lesion and 6 months postsaline, two rats were reanesthetized with chloral hydrate $(400 \mathrm{mg} /$ $\mathrm{kg}$ ) and received in the dorsal column nuclei pressure injections of 100 $\mathrm{nl}$ (over $12 \mathrm{~min}$ ) of a $10 \%$ solution of wheat germ agglutinin conjugated to HRP (WGA-HRP; Sigma) through a $1 \mu$ l Hamilton syringe. The tracer was injected bilaterally, approximately $0.7 \mathrm{~mm}$ lateral to the obex.

Following a postoperative survival time of $48 \mathrm{hr}$, the animals received an overdose of sodium pentobarbital and were perfused transcardially with saline, followed by a phosphate-buffered solution containing $2 \%$ paraformaldehyde, $2 \%$ glutaraldehyde, and $4 \%$ sucrose at $\mathrm{pH} 7.4$. The brains and brainstems were immediately removed and postfixed $2 \mathrm{hr}$ in the same fixative at $4^{\circ} \mathrm{C}$, before being placed in a phosphate-buffered solution containing $30 \%$ sucrose until they sank. Blocks werc then stored at $-80^{\circ} \mathrm{C}$ before further processing.

Cryostat sections ( 20 and $30 \mu \mathrm{m}$ thickness, alternately) were cut serially in the horizontal plane. Sections were treated according to the protocol for the visualization of HRP described by Mesulam (1978) with $3,3^{\prime}, 5,5^{\prime}$-tetramethylbenzidine. Alternate sections were counterstained with neutral red at $\mathrm{pH} 3.5$ before dehydration and final mounting.

The size of the label-containing area was evaluated on $30-\mu \mathrm{m}$-thick sections. Counts were done in both animals each time. The surface of the label-containing area in seven sections, taken at regular intervals over the entire length of the projection area, was measured on a Biocom image analyzer using low-magnification lenses.

Evaluation of the density of the HRP reaction product was done on $20-\mu \mathrm{m}$-thick sections. Because the WGA-HRP staining is punctate and not continuous, actual quantification of axons and terminals could not be obtained. HRP reaction product forms small granules that were therefore counted in order to provide an estimate of the density of labeled fibers and terminals. HRP reaction granules were counted in a $1600 \mu \mathrm{m}^{2}$ square frame using $100 \times$ magnification lenses. This frame was randomly displaced throughout the projection area. Twelve counts were done on three nonadjacent sections for each side in both animals.

Labeling by diffusion of WGA-HRP in axotomized lemniscal axons. Rats were reanesthetized with chloral hydrate $(400 \mathrm{mg} / \mathrm{kg})$ and received a bilateral injection of WGA-HRP in the medial lemniscus using a $1 \mu \mathrm{l}$ Hamilton syringe with a large needle $(0.75 \mathrm{~mm}$ diameter) in order to obtain an axotomy. Twenty to forty nanoliters of the $10 \%$ WGA-HRP solution were injected (over $8 \mathrm{~min}$ ) either just caudal to VB (A 3.7, L 1.5, H 2.2, according to Paxinos and Watson, 1982) at 4, 8, and $21 \mathrm{~d}$ and 3 and 6 months after lesion, or more caudally (A 2.2, L 1, H 0.8) at $15 \mathrm{~d}$ and 1,2 , and 6 months after lesion, in order to label axons in the VB or in the afferent fiber tract, respectively. Two animals were used at each time for each target. They were perfused $15-20 \mathrm{hr}$ after WGA-HRP injection according to the protocol described above.

Cryostat sections ( $48 \mu \mathrm{m}$ thickness) were cut serially in the parasagittal plane. Histochemical revelation of the peroxidase activity was performed in a $0.05 \mathrm{M}$ Tris solution containing $0.05 \% \mathrm{DAB}, 0.9 \%$ nickel ammonium sulfate, and $0.02 \%$ hydrogen peroxide. After $45 \mathrm{~min}$ of incubation, the sections were rinsed in phosphate buffer and then mount- ed onto gelatin-coated slides and allowed to dry overnight. Alternate sections were Nissl stained with cresyl violet before dehydration and final mounting.

\section{Results}

The spread of the neuronal loss on the right side of the brain was observed on sections counterstained with either neutral red (after anterograde transport) or cresyl violet (after labeling by diffusion). The area of neuronal loss always comprised at least the lateral part of VB (DCN projection area) and the reticular nucleus. It extended most often into the medial part of VB (trigeminal projection area), the caudal striatum, and the hippocampal area CA3.

The lesions were characterized by an increased density of nonneuronal cells. Starting 1 month after kainate injection, a progressive decrease in the size of the lesioned areas was observed, related to a shrinkage of the tissue also revealed by a decreased distance between the internal capsule and the midline, and by an enlargement of the lateral ventricle.

\section{Anterograde transport of WGA-HRP from the $D C N$}

For all the cases presented here, it was established that the HRP injection sites encompassed the entire dorsal column nuclei on both sides, in order to ascertain that all DCN projections were labeled. This permitted a quantitative analysis of the results.

Intact $V B$. At low magnification on the intact side, anterogradely transported WGA-HRP indicated a well-delineated projection area, which appeared dark blue as a result of TMB histochemistry. It comprised the lateral part of VB (defined as VPL in the stereotaxic atlas of Paxinos and Watson, 1982), displaying a crescent shape with smooth contours bordered laterally by the internal capsule and the reticular nucleus, medially by the medial part of VB, and ventrocaudally by the medial lemniscus (Fig. 1, left).

At higher magnification in the projection area, labeled afferents, which appeared as a punctate labeling after the TMB histochemistry, were very densely packed (Fig. $2 a$ ). Quantification of $H R P$ reaction granules in the intact $\mathrm{VB}$ revealed no significant change over time (Table 1).

Kainate-lesioned VB. The projection area was still well delineated at all survival times, conserving its shape, anatomical borders, and smooth contours (Fig. 1, right). During the first month after lesion, no change in the extent of the area of projection could be detected (Fig. 1a). A progressive decrease in the DCN projection area was detectable starting 1 month after the lesion (Fig. 1b). Quantification of the size of the label-containing area indicated no decrease on the lesioned side at $9 \mathrm{~d}$ $(L / I \times 100=111$ in one animal, 90 in the other $)$ and $23 \mathrm{~d}(120$, 81 , a decrease of approximately $55 \%$ at 4 months $(42,51)$, and $75 \%$ at 8 months $(27,23)$.

A progressive decrease in the number of HRP reaction granules was observed during the first month in the projection area (Fig. 2b,c; Table 1). At $9 \mathrm{~d}$, the density of labeling was decreased by approximately $35 \%$ relative to the intact side. Further decreases were observed at later times up to an approximate value of $75 \%$ calculated 2 months after the lesion (Fig. $2 d$, Table 1 ). At the same time, morphological alteration of the labeled afferents was apparent, illustrated by the formation of axonal varicosities.

In some cases ( $n=2$ at $9 \mathrm{~d}, 23 \mathrm{~d}, 4$ months), the labeling in the afferent fiber tract and the labeling in the projection area were in the same section (see Fig. $7 a$ ). The afferent fiber tract 


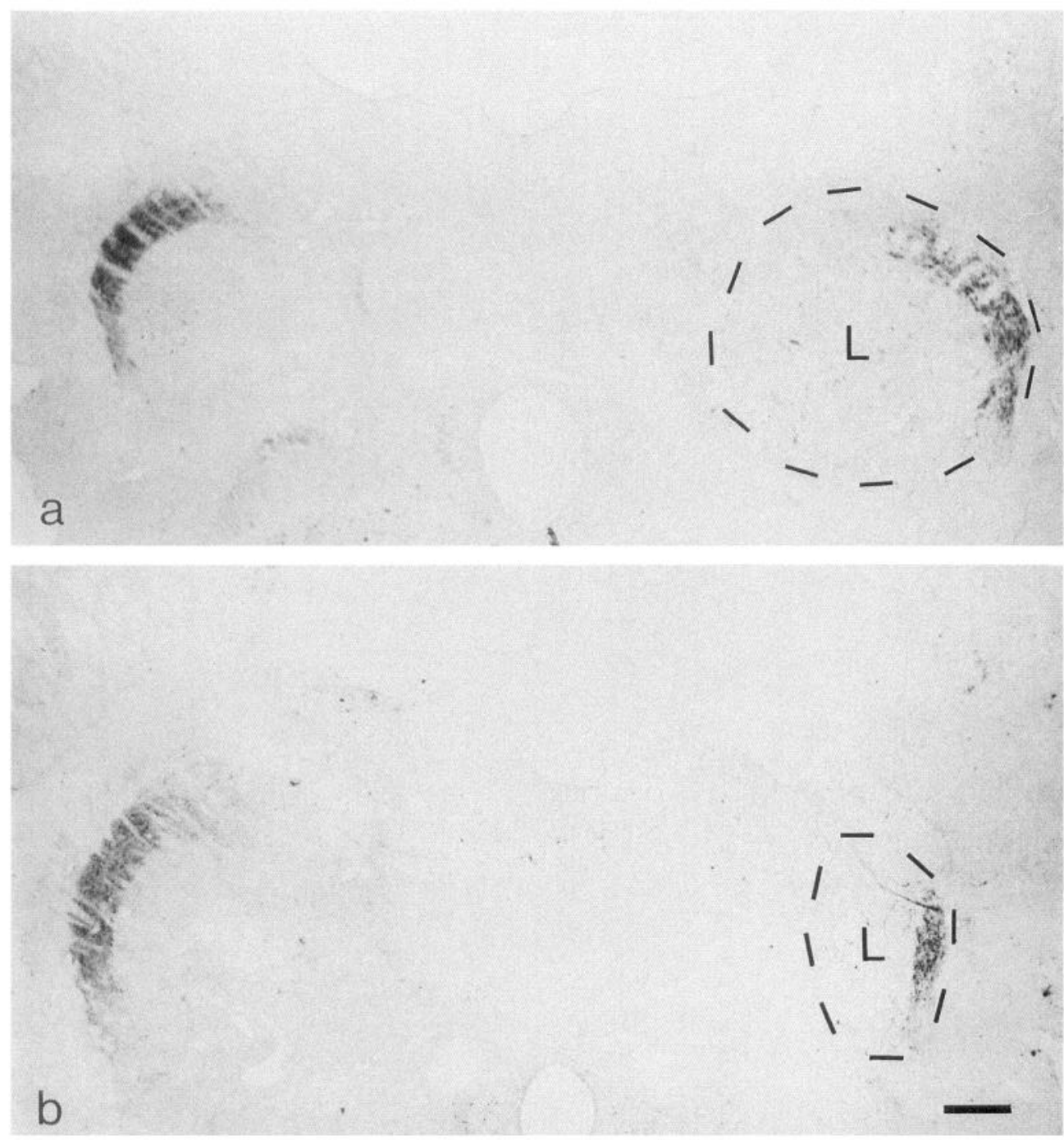

Figure 1. Low-magnification photomicrographs of axonal labeling after anterograde transport of WGA-HRP from the dorsal column nuclei at $23 \mathrm{~d}(a)$ and 8 months $(b)$ postlesion. $L$, lesioned area. Note the decrease in the density of labeling at both times and the shrinkage of the area of projection at 8 months on the lesion side. Scale bar, $500 \mu \mathrm{m}$. (medial lemniscus) runs in a rostrolateral direction as a massive and well-delineated bundle of fibers. Axons enter the projection area in a rostromedial direction. At all of the times studied, it was not possible to detect the presence of varicosities in the fiber tract: the varicosities were confined to the projection area.

Saline-injected animals. Six months after saline injection, no modification of either the size of the projection area, the axonal density, or the morphology of DCN afferents was observed.

\section{Axonal morphology in the thalamus}

In order to visualize axonal morphology both in the projection area and in the afferent fiber tract, axons were labeled from a site of axotomy located at two different points in the medial lemniscus caudal to the thalamus (see Materials and Methods). The more rostral injections permitted the labeling of axons and their terminal arborizations, but the spread of the injection site

Table 1. Quantification of the labeling in the thalamus after anterograde transport of WGA-HRP from the thalamus

\begin{tabular}{|c|c|c|c|c|c|c|c|c|c|c|c|c|c|c|c|c|}
\hline & \multicolumn{2}{|l|}{$4 \mathrm{~d}$} & \multicolumn{2}{|l|}{$9 \mathrm{~d}$} & \multicolumn{2}{|l|}{$12 \mathrm{~d}$} & \multicolumn{2}{|l|}{$23 \mathrm{~d}$} & \multicolumn{2}{|l|}{$2 \mathrm{~m}$} & \multicolumn{2}{|l|}{$4 \mathrm{~m}$} & \multicolumn{2}{|l|}{$6 \mathrm{~m}$} & \multicolumn{2}{|l|}{$8 \mathrm{~m}$} \\
\hline & 1 & 2 & 1 & 2 & 1 & 2 & 1 & 2 & 1 & 2 & 1 & 2 & 1 & 2 & 1 & 2 \\
\hline \multirow[t]{2}{*}{ C } & 260 & 247 & 212 & 270 & $181^{\circ}$ & 257 & 277 & 261 & 274 & 247 & 239 & 249 & 253 & 237 & 213 & 217 \\
\hline & \pm 9 & \pm 7 & \pm 13 & \pm 9 & \pm 7 & \pm 14 & \pm 15 & \pm 7 & \pm 18 & \pm 9 & \pm 22 & \pm 8 & \pm 9 & \pm 7 & \pm 11 & \pm 10 \\
\hline \multirow[t]{2}{*}{$\mathrm{L}$} & 233 & 208 & 150 & 146 & 111 & 148 & 123 & 103 & 67 & 62 & 50 & 57 & 74 & 67 & 84 & 75 \\
\hline & \pm 9 & \pm 13 & \pm 8 & \pm 8 & \pm 9 & \pm 13 & \pm 9 & \pm 9 & \pm 4 & \pm 5 & \pm 4 & \pm 5 & \pm 4 & \pm 8 & \pm 8 & \pm 6 \\
\hline $\mathrm{L}: \mathrm{C}$ & 90 & 84 & 71 & 54 & 61 & 58 & 44 & 45 & 24 & 25 & 21 & 23 & 29 & 28 & 39 & 35 \\
\hline
\end{tabular}
$\times 100$

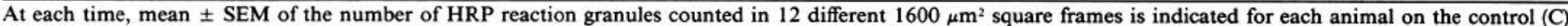

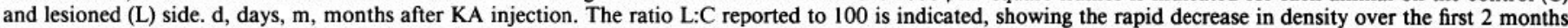
followed by a relative stabilization. 

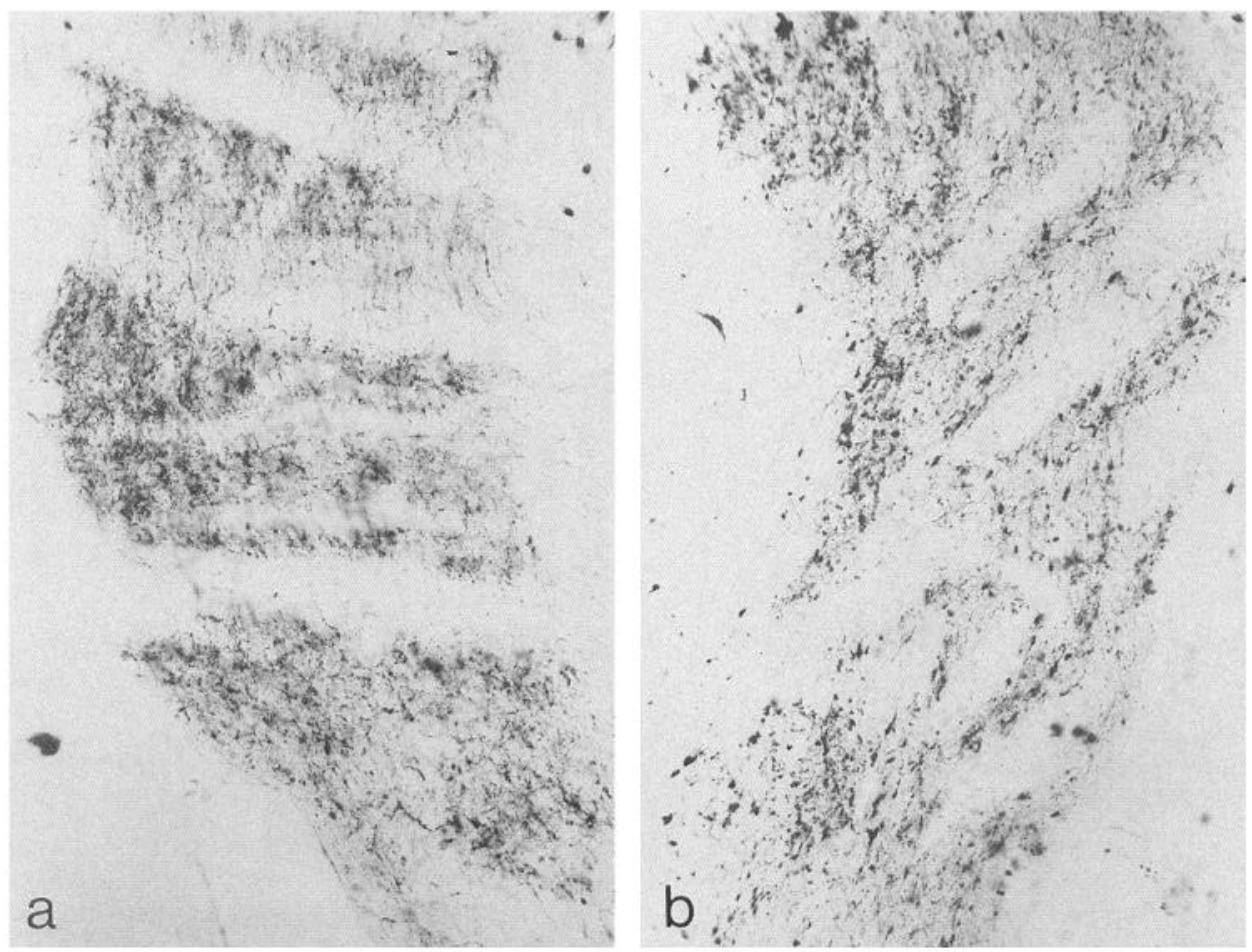

Figure 2. Photomicrographs of axonal labeling after anterograde transport of WGA-HRP from the dorsal column nuclei in the control $(a), 9$-dlesioned $(b), 23$-d-lesioned $(c)$, and 8 -months-lesioned $(d)$ thalamus. Note the decrease in the density of labeling during the first month after lesion. Scale bar, $100 \mu \mathrm{m}$.
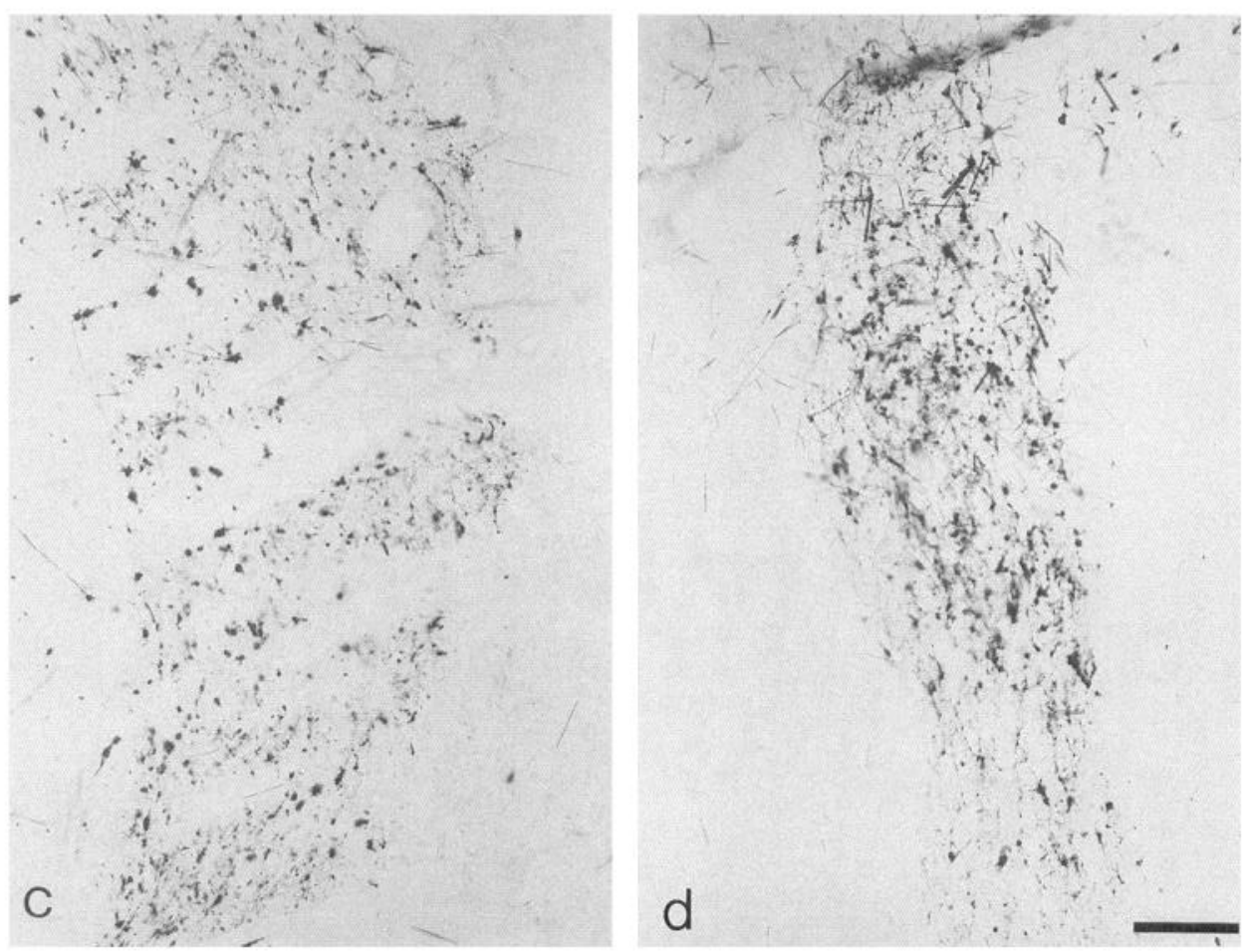

usually did not allow a good appreciation of axonal morphology in the medial lemniscus. Reciprocally, the more caudal injections permitted a good visualization of the axons in the medial lemniscus but the labeling by diffusion did not usually extend into the most terminal portions. In contrast with the results obtained after the DCN injections, the number of labeled axons was quite variable from one animal to another.

Intact side. The diffusely stained lemniscal afferents entered the VB from the medial lemniscus and projected over the entire nucleus (Fig. $3 a$ ). In the projection area, the axons gave off collaterals, commonly at right angles to the main axon, which ramified further (Fig. $3 b$ ). Within the arboreal organization, clusters of terminal swellings, namely, small varicosities within plexus of thin fibers, could be observed [ $C$ ) in Figs. 4-6].

The medial lemniscus appeared as a massive bundle of labeled fibers. Lemniscal axons appeared as thin processes running parallel to the axis of the fiber tract (Fig. $7 a$ ). In all cases a small number of axons presented an enlarged caliber, suggesting de- 

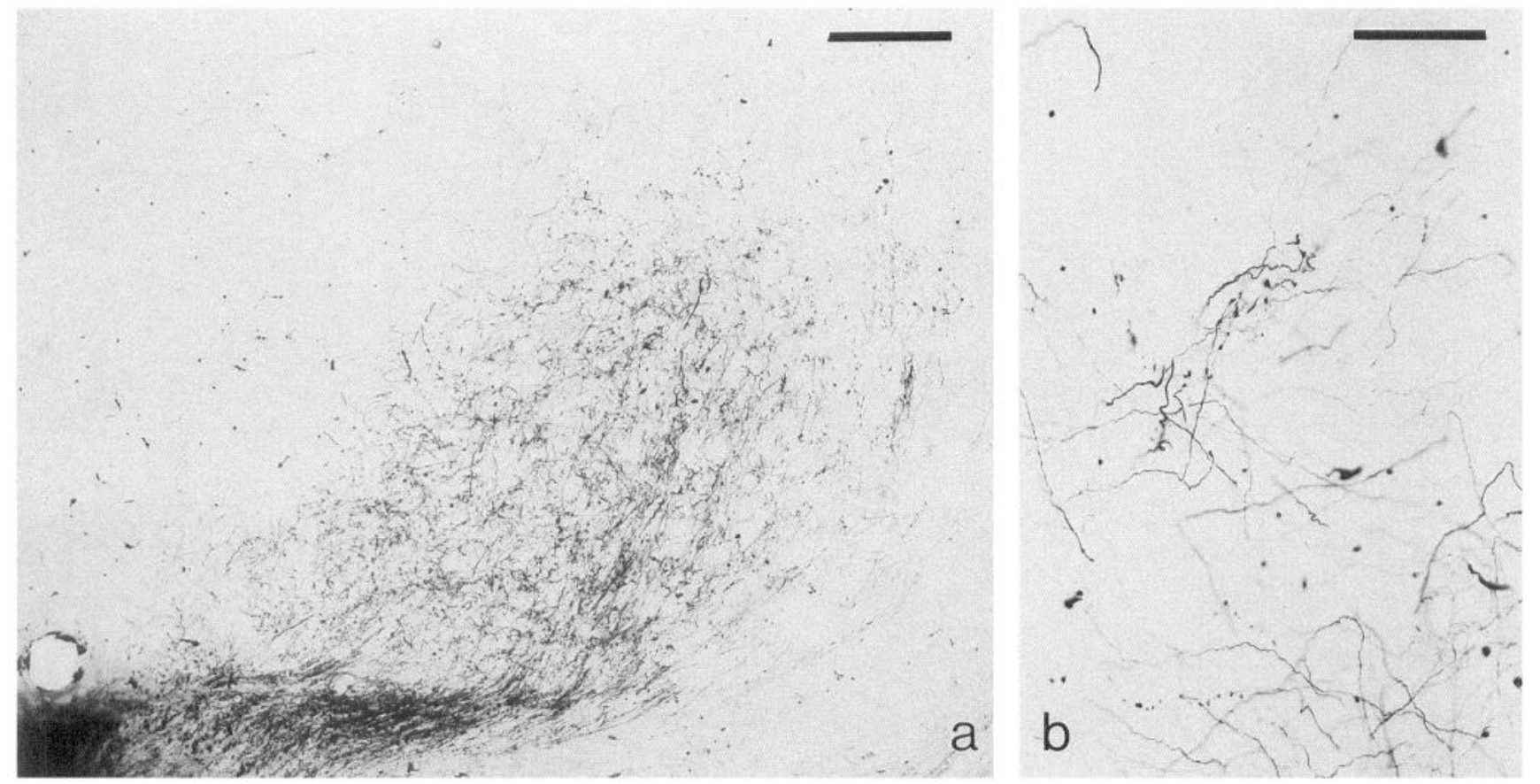

Figure 3. Diffuse axonal labeling using WGA-HRP in the control thalamus. Scale bars: $a, 300 \mu \mathrm{m} ; b, 100 \mu \mathrm{m}$.

generative changes. This picture was encountered near the injection site but very rarely below the thalamic gray matter and was likely due to the experimental procedure.

Kainate-lesioned side. At $4 \mathrm{~d}$ postlesion, no change in axonal arborization was detectable in VB. Axonal terminal arborizations still exhibited a highly ramified aspect with clusters of terminal swellings [Fig. 4 (4)]. In sharp contrast, at $21 \mathrm{~d}$ postlesion, there was an obvious decrease in the density of axonal terminal arborizations [Fig. 4(21)]. The thinner axonal branches were much reduced compared to thicker ones. At 3 months postlesion, axonal arbors in VB were comparable to those observed at $21 \mathrm{~d}$.

At $4 \mathrm{~d}$ postlesion clusters of terminal swellings were still observed. At higher magnification some swellings appeared morphologically different from normal. They were enlarged and crenulated. Although smooth surfaces were still seen, extensions frequently arose from the terminal swellings [Figs. 4 (4), 6 (4d)].

At $8 \mathrm{~d}$ postlesion, some clusters of terminal swellings contained varicosities that could be distinguished by their large size [Fig. $5(8)$ ]. These varicosities had smooth contours, and a round or ovoid shape. Other varicosities could be found on axons, either in a terminal position or along their course in the VB.

At $21 \mathrm{~d}$ postlesion, clusters of terminal swellings were very rare. Numerous large varicosities were observed, either in a terminal position or along the course of the axons in the VB [Figs. 5 (21), 6 (21d)]. They did not form plexuses. The same fiber could bear varicosities separated by a long and apparently unaltered portion of axon.

At 3 and 6 months postlesion, terminal swellings were no longer observed. The axonal varicosities increased in size [Figs. $5(90), 6(3 \mathrm{~m}, 6 \mathrm{~m})]$.

In the medial lemniscus, the morphology of axons was similar to that on the intact side at all times (Fig. 7b). In particular, no axonal varicosity was observed.

\section{Discussion}

Anterograde transport of WGA-HRP showed a continuous decrease of the number of axonal elements in the lesion. For the quantitative analysis of these results, it was first established that the HRP injection sites encompassed the entire dorsal column nuclei on both sides, in order to ascertain that all DCN projections were labeled. It was not possible to avoid slight variations in the intensity of histochemical staining from one case to another. To limit the bias introduced by this variability, the values obtained on the lesioned sides were expressed as percentages of the values on the control side. During the first month after lesion, the decrease in number of axonal elements resulted in a decrease in the density of labeling in the lesion, without obvious shrinkage in the volume of the DCN projection area. After 1 month, in contrast, the continuing decrease in the number of axonal elements in the lesion resulted in the shrinkage of the projection area while axonal density remained constant. This long-term decrease, however, progressed much more slowly than during the first month, and numerous labeled elements were still observed after 8 months.

This decrease in the number of axonal elements in the lesion could be explained by any of three phenomena: (1) a decrease in terminal arborization of axons in the area of neuronal loss, (2) a retraction of axons, or (3) a death of neurons in the DCN resulting in anterograde degeneration of afferent axons. Our morphological data indicate that the first mechanism, that is, dramatic loss of axonal terminal arborizations of DCN projections, occurs during the first month after ablation of VB target neurons. This loss of axonal branches is accompanied by a loss of terminal swellings and by the formation of large axonal varicosities. The absence of such varicosities in the afferent fiber tract suggests that the loss of axonal terminal arborizations is not followed by a retraction of axonal stems from the neuron- 


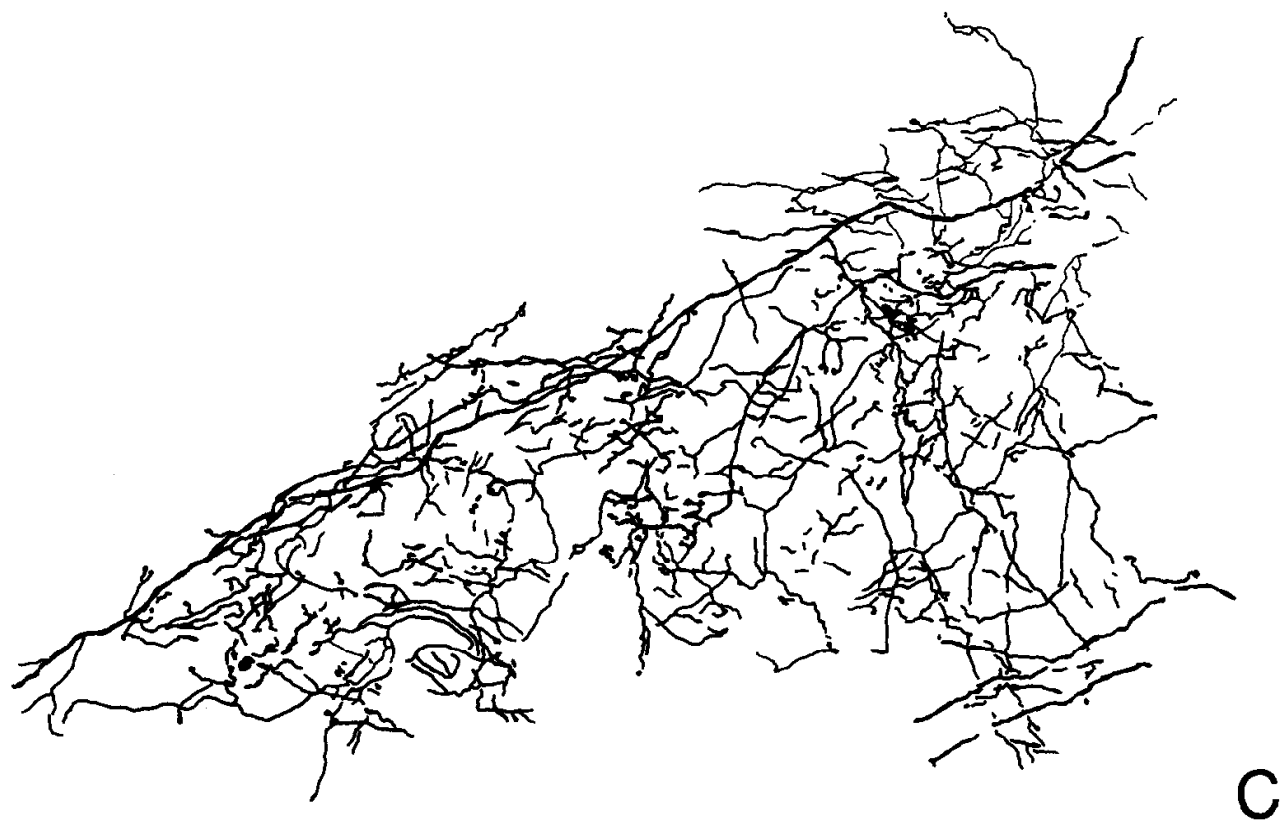

Figure 4. Camera lucida drawings, using low-magnification lenses, of lemniscal axonal arborization stained by diffuse labeling using WGA-HRP in the control $(C)$, 4-d-lesioned (4), and 21d-lesioned $(2 I)$ thalamus. Note the similarity between the results obtained in the control and in the 4-d-lesioned thalamus, compared to the sparser arborization at $21 \mathrm{~d}$ postlesion. Scale bar, $50 \mu \mathrm{m}$.

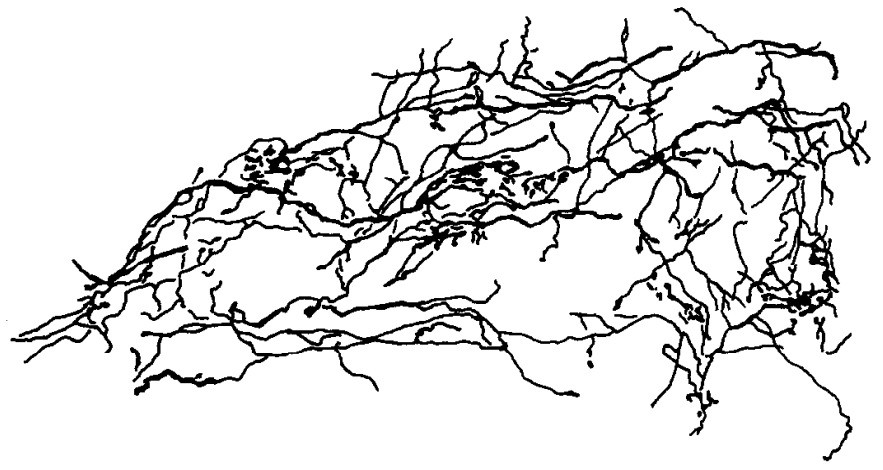

4
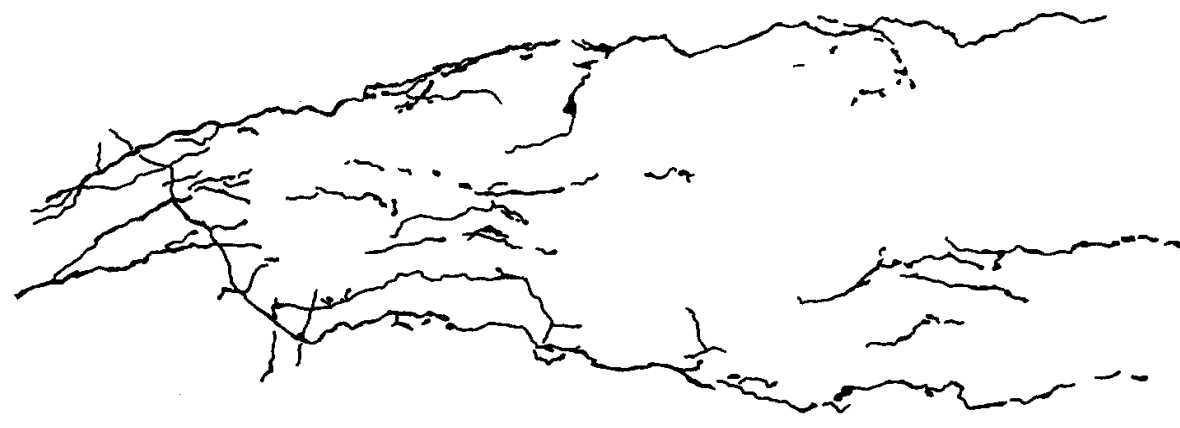

21 depleted area, while the long-term maintenance of axons in the lesion indicates that neuronal death in the DCN, if it occurs, is partial.

The first month postlesion is characterized by a strong decrease in axonal density in the lesion, as revealed by the decrease in the density of HRP reaction granules following anterograde transport from the DCN. Our study shows that this decrease can be explained by the morphological alteration of axons that occurs during this period in the neuron-depleted VB. There is indeed a loss of axonal terminal arborizations leading to a simplification of axonal arbors, in parallel with the appearance of axonal varicosities. The reduction of the proportion of thin versus thick axonal branches is also an indication of the simplification of axonal terminal arborizations. More direct evi- 

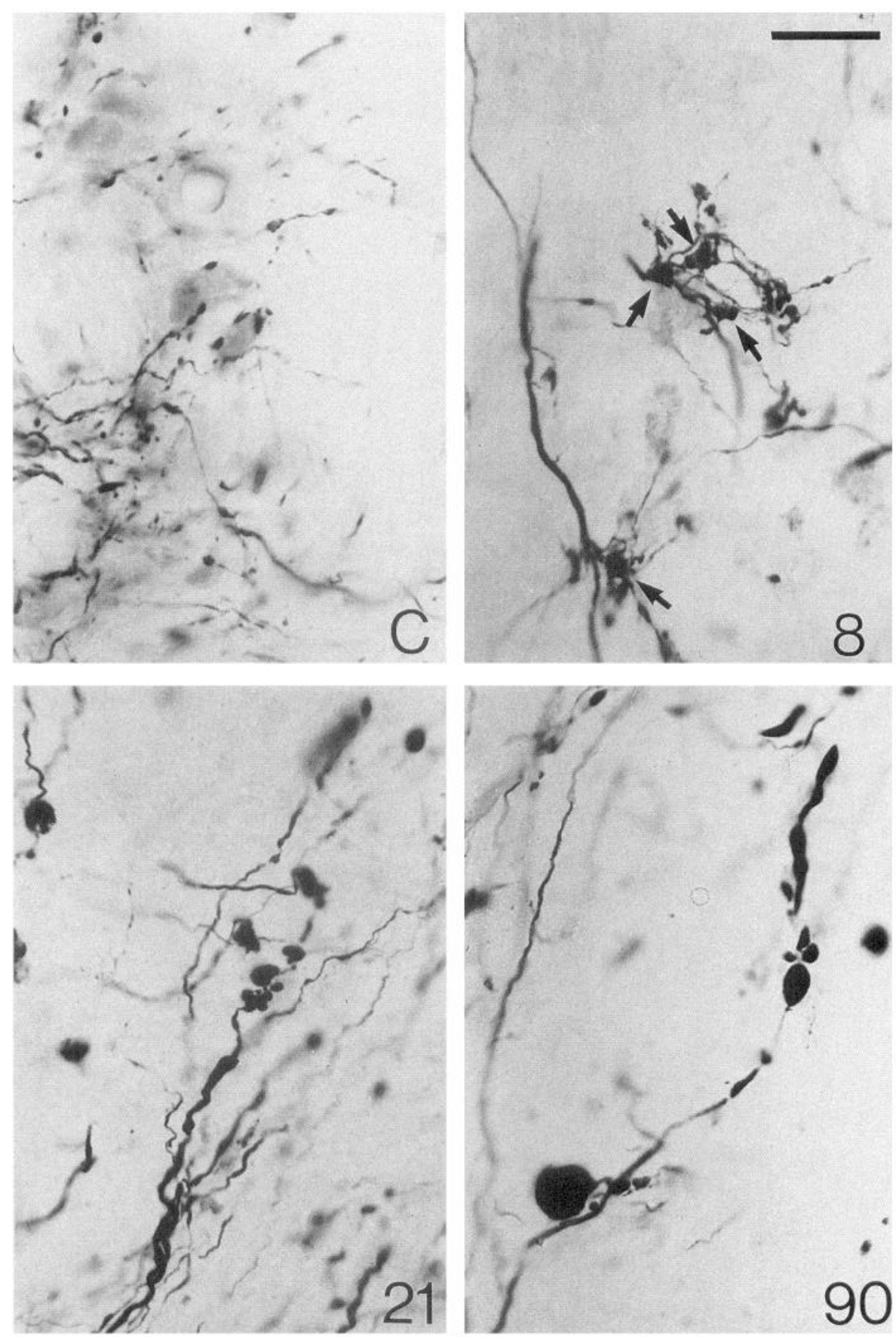

Figure 5. High-magnification photomicrographs of axonal terminals and varicosities stained by diffuse labeling using WGA-HRP on the control side $(C)$ and 8,21 , and $90 \mathrm{~d}$ after $\mathrm{KA}$ injection. Note that plexuses of fine axonal branches have disappeared at 21 $\mathrm{d}$ postlesion. Axonal varicosities are already present at $8 \mathrm{~d}$ postlesion (arrows), and increase in size at later times. Scale bar, $20 \mu \mathrm{m}$. dence of terminal loss is found in the observation of a disappearance of the plexuses of terminal swellings. This indicates that a reduction of lemniscal axonal terminal arborizations takes place after excitotoxic loss of their VB target cells. This result is consistent with those of Armengol et al. (1989) and Rossi et al. (1993) on climbing fibers afferent to the excitotoxically lesioned cerebellum, suggesting a general phenomenon in the CNS.

The mechanisms at work in this process are unclear. They may be related to (1) a direct effect of the excitotoxin, (2) an indirect effect not related to the target loss itself but to glial scarring, or (3) the loss of target cells. A direct effect of KA is unlikely. Studies on excitotoxic lesions indicate an absence of morphological alteration of afferents during the first days fol- lowing the lesion (Herndon and Coyle, 1977; Coyle et al., 1978; Pearson et al., 1991). In our case, terminal arborizations were still observed at $8 \mathrm{~d}$ postlesion. It has been postulated that large axonal varicosities appearing after excitotoxic lesions may indicate a direct effect of KA on afferent axons (Meibach et al., 1978; Walker and McAllister, 1986). Axonal varicosities have been described after KA lesions in various parts of the brain (Peschanski and Besson, 1987; Nothias et al., 1988; Pearson et al., 1992). In all these cases, however, they were not observed during the first days following the lesion, and could thus be secondary to the target loss. Furthermore, Rossi et al. (1993) observed a disappearance of terminal axonal branches of climbing fibers when Purkinje cells were destroyed using propidium 

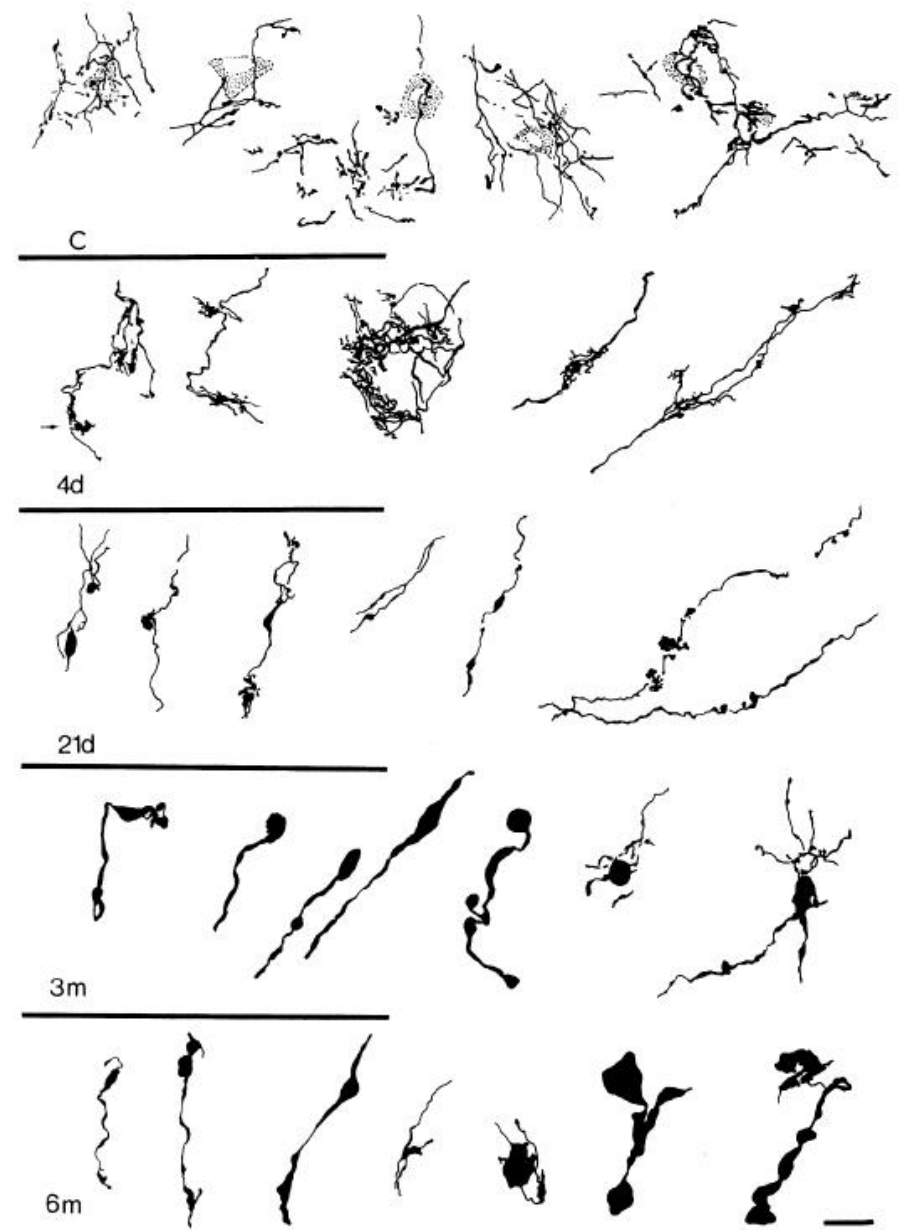

Figure 6. Camera lucida drawings, using high-magnification lenses, of axonal terminals and varicosities in the control $(C), 4$-d-lesioned $(4 d)$, 21-d-lesioned $(21 d)$, 3-month-lesioned $(3 \mathrm{~m})$, and 6-month-lesioned $(6 \mathrm{~m})$ iodide instead of KA. Since the mechanisms of neuronal death are different for these two toxins, one can suppose that the loss of axonal branches is not related to a direct effect of the excitotoxin.

Changes in glial populations after the excitotoxic lesion could also influence the fate of afferents. The kainate-lesioned VB is characterized by a massive recruitment and proliferation of microglial/macrophagic cells during the first month after lesion, in the absence of reactive astrocytes (Dusart et al., 1991; Marty et al., 1991). Studies performed during development (Innocenti et al., 1983) support the hypothesis that activated microglia and macrophages are able to eliminate axonal branches. On the other hand, axonal changes may be due to a progressive retraction of terminal branches due to target loss. This hypothesis is supported by the fact that varicosities are first seen associated with the clusters of terminals, suggesting a mechanism starting at the terminals and progressing retrogradely. Our result is consistent with the finding of Rossi et al. (1993) that the loss of climbing fiber arborizations after an excitotoxic lesion of the cerebellum progresses retrogradely. Varicosities could then reflect the accumulation of axoplasmic elements within a retracted portion of the axon.

Axonal arbors could be dependent on the continuous release of trophic substances by target cells, or on contacts with those targets, or both. Trophic factors are synthetized in a regulated manner by neurons in the adult CNS (Castrén et al., 1992) and adult neurons possess high-affinity receptors for those factors (Merlio et al., 1992). They may, therefore, play a role in trophic interactions between afferent neurons and their targets. Although survival of afferent neurons does not strictly depend upon trophic factors (Sofroniew et al., 1990; Kordower et al.,

thalamus. In the control, Nissl-stained neurons are indicated by stipples. Scale bar, $20 \mu \mathrm{m}$.
Figure 7. Axonal labeling after anterograde transport of WGA-HRP from the dorsal column nuclei at $23 \mathrm{~d}$ postlesion $(a)$ and diffuse labeling at 6 months $(b)$. Note that axonal varicosities are not found in the afferent fiber tract (medial lemniscus, $m l$ in $a$, arrows in $b$ ). Scale bar: $300 \mu \mathrm{m}$ for $a, 95 \mu \mathrm{m}$ for $b$.
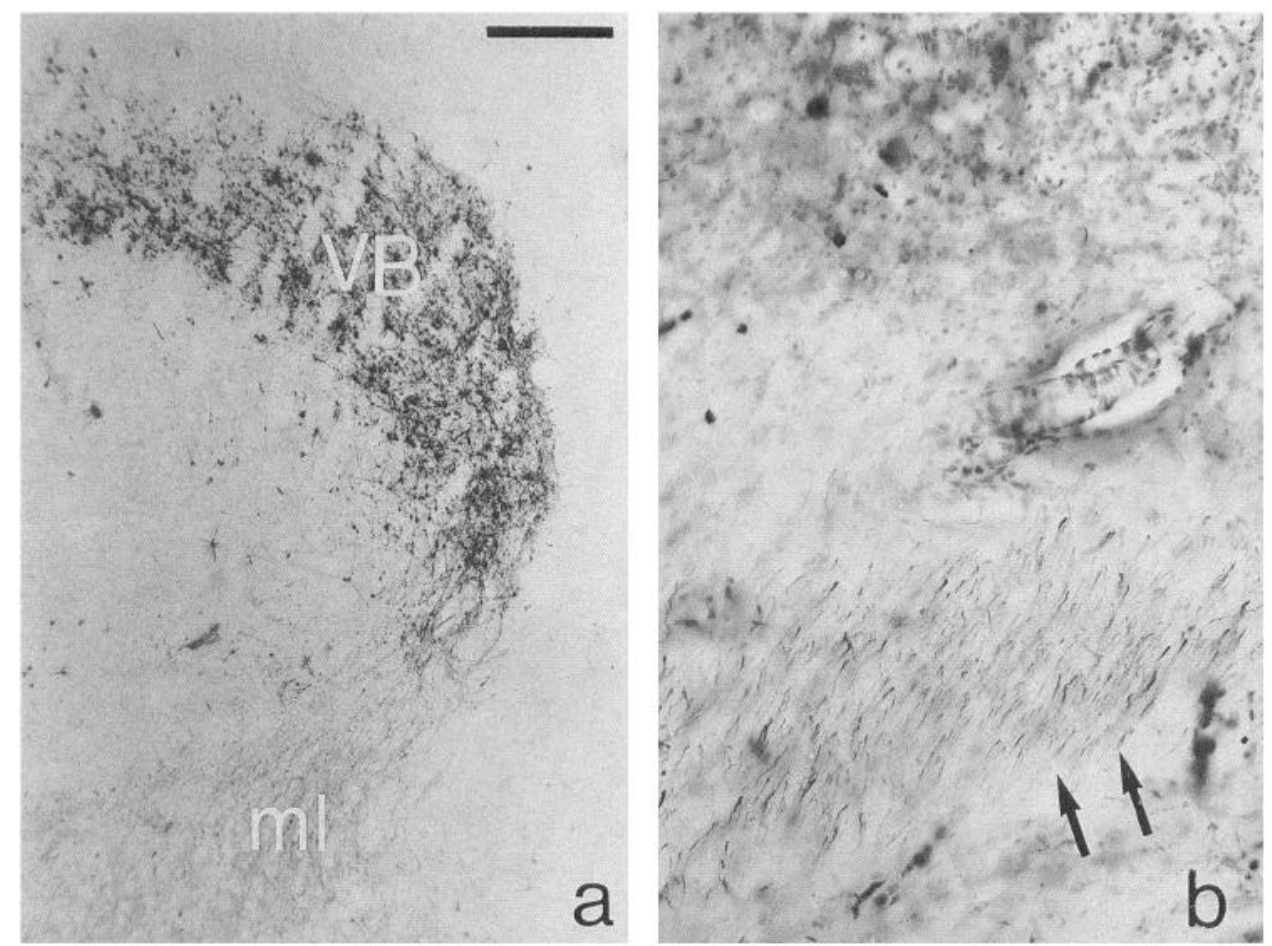
1992) as it does during development, it has been shown in vitro that trophic factors could act differentially on axonal arborization and neuronal survival (Campenot, 1977). This role on the terminal axonal compartment may dominate in the adult. Van Der Zee and co-workers (1992) showed that neurotrophins are necessary for deafferentation-induced extension of axonal arbors in the adult CNS. One may speculate that trophic factors released by VB neurons would play a tonic role in the maintenance of the axonal arborization of DCN projections. Experiments are presently in progress in our laboratory to reveal growth factors that may be active in such a hypothetical process (Junier and Peschanski, 1992). Another possibility is that the simple loss of contacts between afferents and their targets is sufficient to induce a retraction of axonal branches. Rossi et al. (1993) propose that loss of a contact factor on Purkinje cells might be responsible for the loss of climbing fibers arborization. Their hypothesis is supported by the fact that after grafting or deafferentation-induced sprouting, climbing fibers arborize only when they contact Purkinje cells. Whether this is also the case in our system remains to be shown.

One month after the lesion, the decrease in the number of axons in the lesion continues, as indicated by a shrinkage of the projection area while axonal density is stabilized. This could be explained either by a continuing axonal retraction or by a neuronal death in the DCN followed by the anterograde degeneration of axons. A long-term continuous retraction of axons was not observed in climbing fibers after excitotoxic lesion of Purkinje cells (Rossi et al., 1993). In our experiments, the lack of morphological changes of axons in the medial lemniscus after the lesion (which clearly contrasted with the appearance of axonal varicosities in the terminal area as early as $8 \mathrm{~d}$ postlesion) is also an argument against this possibility. Axonal varicosities were confined to the lesion site or its borders. This suggests that the varicosities are not retraction bulbs, as described after axotomy by Ramon y Cajal (1928), since they would have progressed backward along the axons. This agrees with previous data at the electron microscopic level, showing that at 1 month postlesion most of these varicosities do not present morphological characteristics of degeneration (Peschanski and Besson, 1987). Alternatively, however, one cannot exclude that retraction may occur in the absence of retraction bulbs. In conclusion, the most likely explanation for the decrease in the number of axons many months after the lesion is the death of DCN neurons followed by the anterograde degeneration of their axon. This hypothesis of a neuronal death in the DCN following thalamic target deprivation is presently under investigation in our laboratory. Since axons are still numerous in the 8 months lesion, such neuronal death would, in any case, be partial.

\section{References}

Armengol JA, Sotelo C, Angault P, Alvarado-Mallart RM (1989) Organization of host afferents to cerebellar grafts implanted into the kainate lesioned cerebellum in adult rats. Hodological evidence for the specificity of host-graft interactions. Eur J Neurosci 1:75-93.

Campenot RB (1977) Local control of neurite development by nerve growth factor. Proc Natl Acad Sci USA 74:4516-4519.

Castrén E, Zafra F, Thoenen H, Lindholm D (1992) Light regulates expression of brain-derived neurotrophic factor mRNA in rat visual cortex. Proc Natl Acad Sci USA 89:9444-9448.

Cowan WM, Fawcett JW, O'Leary DDM, Stanfield BB (1984) Regressive events in neurogenesis. Science 225:1258-1265.

Coyle JT, Schwarcz R (1983) The use of excitatory amino acids as selective neurotoxins. In: Handbook of chemical neuroanatomy, Vol 1 (Björklund A, Hökfelt T, eds), pp 508-527. Amsterdam: Elsevier.
Coyle JT, Molliver ME, Kuhar MJ (1978) In situ injection of kainic acid: a new method for selectively lesioning neuronal cell bodies while sparing axons of passage. J Comp Neurol 180:301-324.

Divac I, Markowitsch HJ, Pritzel M (1978) Behavioral and anatomical consequences of small intrastriatal injections of kainic acid in the rat. Brain Res 151:523-532.

Dusart I, Marty S, Peschanski M (1991) Glial changes following an excitotoxic lesion in the CNS. II. Astrocytes. Neuroscience 45:541549.

Herndon RM, Coyle JT (1977) Selective destruction of neurons by a transmitter agonist. Science 198:71-72.

Innocenti GM, Clarke S, Koppel H (1983) Transitory macrophages in the white matter of the developing visual cortex. II. Development and relations with axonal pathways. Dev Brain Res 11:55-66.

Isacson O, Brundin P, Gage FH, Björklund A (1985) Neural grafting in a rat model of Huntington's disease: progressive neurochemical changes after neostriatal ibotenate lesions and striatal tissue grafting. Neuroscience 16:799-817.

Junier M-P, Peschanski M (1992) Induction of neurotrophic factors gene expression in the kainic acid lesioned thalamus. Soc Neurosci Abstr 18:427.

Kordower JH, Burke-Watson M, Roback JD, Wainer BH (1992) Stability of septohippocampal neurons following excitotoxic lesions of the rat hippocampus. Exp Neurol 117:1-16.

Marty S, Dusart I, Peschanski M (1991) Glial changes following an excitotoxic lesion in the CNS. I. Microglia/macrophages. Neuroscience 45:529-539.

Meibach RC, Brown L, Brooks FH (1978) Histofluorescence of kainic acid-induced striatal lesions. Brain Res 148:219-223.

Merlio JP, Ernfors P, Jaber M, Persson H (1992) Molecular cloning of rat trkC and distribution of cells expressing messenger RNAs for members of the trk family in the rat central nervous system. Neuroscience 51:513-532.

Mesulam MM (1978) Tetramethyl benzidine for horseradish peroxidase neurohistochemistry: a noncarcinogenic blue reaction product with superior sensitivity for visualizing neural afferents and efferents. J Histochem Cylochem 26:106-117.

Nothias F, Wictorin K, Isacson O, Björklund A, Peschanski M (1988) Morphological alteration of thalamic afferents in the excitotoxically lesioned striatum. Brain Res 461:349-354.

Paxinos G, Watson C (1982) The rat brain in stereotaxic coordinates. Sidney: Academic.

Pearson HE, Stoffler DJ (1992) Retinal ganglion cell degeneration following loss of postsynaptic target neurons in the dorsal lateral geniculate nucleus of the adult cat. Exp Neurol 116:163-171.

Pearson HE, Sonstein WJ, Stoffler DJ (1991) Selectivity of kainic acid as a neurotoxin within the dorsal lateral geniculate nucleus of the cat: a model for transneuronal retrograde degeneration. J Neurocytol 20: 376-386.

Pearson HE, Stoffler DJ, Sonstein WJ (1992) Response of retinal terminals to loss of postsynaptic target neurons in the dorsal lateral geniculate nucleus of the adult cat. J Comp Neurol 315:333-343.

Peschanski M, Besson .IM (1987) Structural alteration and possible growth of afferents after kainate lesion in the adult thalamus. J Comp Neurol 258:185-203.

Ramon y Cajal S (1928) Degeneration and regeneration of the nervous system. Reprint (DeFelipe J, Jones EG, eds). New York: Oxford UP, 1991.

Rossi F, Borsello T, Vaudano E, Strata P (1993) Regressive modifications of climbing fibres following Purkinje cell degeneration in the cerebellar cortex of the adult rat. Neuroscience 53:759-778.

Sofroniew MV, Isacson O (1988) Distribution of degeneration of cholinergic neurons in the septum following axotomy in different portions of the fimbria-fornix: a correlation between degree of cell loss and proximity of neuronal somata to the lesion. J Chem Neuroanat 1:327337.

Sofroniew MV, Galletly NP, Isacson O, Svendsen CN (1990) Survival of adult basal forebrain cholinergic neurons after loss of target neurons. Science 247:338-342.

Van Der Zee CEEM, Fawcett J, Diamond J (1992) Antibody to NGF inhibits collateral sprouting of septohippocampal fibers following entorhinal cortex lesion in adult rats. J Comp Neurol 326:91-100.

Villegas-Pérez MP, Vidal-Sanz M, Rasminski M, Bray GM, Aguayo AJ (1993) Rapid and protracted phases of retinal ganglion cell loss follow axotomy in the optic nerve of adult rats. J Neurobiol 24:23-36. 
Walker PD, McAllister JP II (1986) Anterograde transport of horseradish peroxidase in the nigrostriatal pathway after neostriatal kainic acid lesions. Exp Neurol 93:334-347.

Wictorin K, Fischer W, Isacson O, Nothias F, Peschanski M, Björklund A (1988) Connectivity of striatal grafts implanted into the ibotenic acid lesioned striatum. I. Subcortical afferents. Neuroscience 27:547562.

Zaczek R, Schwarcz R, Coyle JT (1978) Long-term sequelae of striatal kainate lesion. Brain Res 152:626-632. 